\title{
ON VAFA'S THEOREM FOR TENSOR CATEGORIES
}

\author{
PAVEl Etingof
}

\section{Introduction}

In this note we prove two main results.

1. In a rigid braided finite tensor category over $\mathbb{C}$ (not necessarily semisimple), some power of the Casimir element and some even power of the braiding is unipotent.

2. In a (semisimple) modular category, the twists are roots of unity dividing the algebraic integer $D^{5 / 2}$, where $D$ is the global dimension of the category (the sum of squares of dimensions of simple objects).

Both results generalize Vafa's theorem ([V, AM, BaKi]), saying that in a modular category twists are roots of unity, and square of the braiding has finite order.

In the case when the category admits a fiber functor, i.e. is a category of representations of a finite dimensional Hopf algebra, these results can be found in [EG1]. In fact, the method of proof of 1 and 2 is parallel to the proof of Theorems 4.3 and 4.8 in [EG1], modulo two new ingredients: categorical determinants and Frobenius-Perron dimensions.

We note that statement 1 in the semisimple case was proved in [Da1], also using determinants.

At the end of the note, we discuss the notion of the quasi-exponent of a finite rigid tensor category, which is motivated by results 1 and 2 and the paper [EG3].

\section{Determinant of an automorphism}

Let $A$ be a ring, isomorphic to $\mathbb{Z}^{N}$ as an abelain group. By a dimension function on $A$ we mean any nonzero ring homomorphism $d: A \rightarrow \mathbb{C}$. It is clear that values of $d$ are algebraic integers, since $d(X)$ is an eigenvalue of the matrix of left multiplication by $X$ in $A$ in some basis, which has integer entries.

Let $\mathcal{C}$ be a tensor category over $\mathbb{C}$, i.e. a $\mathbb{C}$-linear abelian category with a biexact tensor product functor and associativity isomorphism satisfying the pentagon identity. In this paper we will assume that $\mathcal{C}$ is finite, i.e. equivalent, as an abelain category, to the category of finite dimenisional representations of a finite dimensional $\mathbb{C}$-algebra.

Let $\mathcal{G}(\mathcal{C})$ be the Grothendieck ring of $\mathcal{C}$. It is a free abelian group of finite rank, whose basis $S$ is the set of isomorphsim classes of simple objects of $\mathcal{C}$, and multiplication is given by $X Y=\sum_{Z \in S}[X \otimes Y: Z] Z$, where $[M: Z]$ is

Received July 1, 2002. 
the multiplicity of occurence of $Z$ in $M$ (it is well defined by the Jordan-Hölder theorem).

Now suppose that $\mathcal{G}(\mathcal{C})$ has been equipped with a dimension function $d$. Let $\mathbb{A}$ be the ring of algebraic integers. Then to every automorphism $a: X \rightarrow X$ of an object $X \in \mathcal{C}$ we associate its determinant, which is an element of the $\mathbb{A}$-module $\mathbb{A} \otimes_{\mathbb{Z}} \mathbb{C}^{*}$, by the formula: $\operatorname{det}(a)=\sum_{Z \in S} d(Z) \otimes \operatorname{det}\left(\left.a\right|_{\operatorname{Hom}(P(Z), X)}\right)$, where $P(Z)$ is the projective cover of $Z$. We will use a more convenient multiplicative notation in which $d \otimes c \in \mathbb{A} \otimes \mathbb{C}^{*}$ will be written as $c^{d}$. In this notation, $\operatorname{det}(a)=\prod_{Z \in S} \operatorname{det}\left(\left.a\right|_{\operatorname{Hom}(P(Z), X)}\right)^{d(Z)}$. For example, if $\mathcal{C}=\operatorname{Rep}(\mathrm{H})$, where $H$ is a finite dimensional bialgebra, and $d(Z)=\operatorname{dim}(Z)$, then det takes values in $\mathbb{Z} \otimes_{\mathbb{Z}} \mathbb{C}^{*}=\mathbb{C}^{*} \subset \mathbb{A} \otimes_{\mathbb{Z}} \mathbb{C}^{*}$, and coincides with the usual determinant.

The properties of det are summarized in the following proposition.

\section{Proposition 2.1.}

1) $\operatorname{det}(a b)=\operatorname{det}(a) \operatorname{det}(b)$.

2) If $0 \rightarrow X \rightarrow Y \rightarrow Z \rightarrow 0$ is an exact sequence respected by $a: Y \rightarrow Y$ then $\operatorname{det}\left(\left.a\right|_{Y}\right)=\operatorname{det}\left(\left.a\right|_{X}\right) \operatorname{det}\left(\left.a\right|_{Z}\right)$.

3) If $a$ is a scalar then $\operatorname{det}\left(\left.a\right|_{X}\right)=a^{d(X)}$.

4) $\operatorname{det}\left(\left.\left.1\right|_{X} \otimes a\right|_{Y}\right)=\operatorname{det}\left(\left.a\right|_{Y}\right)^{d(X)}$.

Proof.

- 1) is obvious.

- 2) follows from the fact that for a projective object $P$ the functor $\operatorname{Hom}(P, *)$ is exact.

- 3) follows from the fact that one has $\operatorname{dim} \operatorname{Hom}(P(Z), X)=[X: Z]$.

- Now, 2) implies that it suffices to check 4) for simple $Y$, in which case it is clear from 3) and multiplicativity of $d$, since $a$ is a scalar.

Remark. The determinant of an automorphism is the specialization of the class of this automorphism (discussed in [Da1]) with respect to the dimension function $d$.

\section{Frobenius-Perron dimensions}

Recall that a finite $\mathbb{Z}_{+}$-ring is a ring $A$ (free as a $\mathbb{Z}$-module) with a fixed finite basis $S$, such that the structure constants are nonnegative. For instance, if $\mathcal{C}$ is a finite tensor category, then $\mathcal{G}(\mathcal{C})$ is a finite $\mathbb{Z}_{+}$-ring.

We will say that $A$ is transitive if for any $X, Z \in S$ there exists $Y_{1}, Y_{2} \in S$ such that $X Y_{1}$ and $Y_{2} X$ involve $Z$ with a nonzero coefficient. For example, if $\mathcal{C}$ as above is a rigid monoidal category, then $\mathcal{G}(\mathcal{C})$ is transitive.

For transitive finite $\mathbb{Z}_{+}$-rings, there exists a remarkable dimension function $d_{+}$, called the Frobenius-Perron dimension. (For the theory of this dimension, see $[\mathrm{ENO}]$; the commutative case is discussed in $[\mathrm{FK}]$ and $[\mathrm{AB}]$ ). 
Define the group homomorphism $d_{+}: A \rightarrow \mathbb{C}$ as follows: for $X \in S$, let $d_{+}(X)$ be the maximal nonnegative eigenvalue of the matrix of multiplication by $X$. It exists by the Frobenius-Perron theorem, since this matrix has nonnegative entries.

\section{Proposition 3.1.}

- 1) $d_{+}$is a ring homomorphism, and for any $X \in S, d_{+}(X)>0$.

- 2) There exists a unique, up to scaling, element $R \in A_{\mathbb{C}}$ such that $X R=$ $d_{+}(X) R, X \in A$. After an appropriate normalization this element has positive coefficients, and satisfies $d_{+}(R)>0$ and $R Y=d_{+}(Y) R, Y \in A$.

Proof. Consider the matrix $M$ of right multiplication by $\sum_{X \in S} X$ in $A$ in the basis $S$. By transitivity, this matrix has strictly positive entries, so by the Frobenius-Perron theorem it has a unique up to scaling eigenvector $R \in A_{\mathbb{C}}$ with eigenvalue $\lambda_{M}$ (the maximal positive eigenvalue of $M$ ). Furthermore, this eigenvector can be normalized to have positive entries.

Since $R$ is unique, it satisfies the equation $X R=d(X) R$ for some function $d$ : indeed, $X R$ is also an eigenvector of $M$ with eigenvalue $\lambda_{M}$, so it must be proportional to $R$. Furthermore, it is clear that $d$ is a dimension function. Since $R$ has positive entries, $d(X)=d_{+}(X)$ for $X \in S$. This implies 1$)$. We also see that $d_{+}(X)>0$ for $X \in S$ (as $R$ has positive coefficients), and hence $d_{+}(R)>0$.

Now, by transitivity, $R$ is the unique, up to scaling, solution of the system of linear equations $X R=d_{+}(X) R$ (as the matrix $M^{\prime}$ of left multiplication by $\sum_{X \in S} X$ also has positive entries). Hence, $R Y=d^{\prime}(Y) R$ for some dimension function $d^{\prime}$. Applying $d_{+}$to both sides and using that $d_{+}(R)>0$, we find $d^{\prime}=d_{+}$, as desired.

The element $R$ will be called the regular object, because in the case of $\mathcal{C}=$ $\operatorname{Rep}(\mathrm{H})$ for a Hopf algebra $H$, it is indeed the class of the regular representation of $H$. More generally, we have the following proposition.

Proposition 3.2. If $\mathcal{C}$ is rigid then $R=\sum_{X \in S} d_{+}(X) P(X)$ (up to scaling).

Proof. We have $\sum_{X} d_{+}(X) \operatorname{dim} \operatorname{Hom}(P(X), Z)=d_{+}(Z)$ for any object $Z$. Hence,

$$
\begin{gathered}
\sum_{X} d_{+}(X) \operatorname{dim} \operatorname{Hom}(P(X) \otimes Z, Y)= \\
\sum_{X} d_{+}(X) \operatorname{dim} \operatorname{Hom}\left(P(X), Y \otimes Z^{*}\right)=d_{+}(Y) d_{+}\left(Z^{*}\right)=d_{+}(Y) d_{+}(Z) .
\end{gathered}
$$

Now, $P(X) \otimes Z$ are projective objects. Hence, the formal sums $\sum_{X} d_{+}(X) P(X) \otimes$ $Z$ and $d_{+}(Z) \sum_{X} d_{+}(X) P(X)$ are linear combinations of $P(Y), Y \in \mathcal{C}$, with the same coefficients.

In the sequel, for rigid categories we will use the normalization of $R$ given by Proposition 3.2. In this case, generalizing [ENO], we call the number $d_{+}(R)$ the Frobenius-Perron dimension of $\mathcal{C}$. 
Remark. Propositions 3.1(2) and 3.2 for semisimple rigid categories appear in [Da2].

\section{Generalization of Vafa's theorem}

Recall that a braiding on $\mathcal{C}$ is a functorial isomorphism $\beta: \otimes \rightarrow \otimes^{o p}$ satisfying the hexagon relation. Recall also that in a braided rigid tensor category there exists an automorphism $z$ of the identity functor, called the Casimir element, such that

$$
\left.z\right|_{X \otimes Y}=\left(\left.\left.z\right|_{X} \otimes z\right|_{Y}\right)\left(\beta^{2}\right)^{-2} .
$$

Namely, let ${ }^{*} X, X^{*}$ denote the left and right duals to $X$. Then $\left.z\right|_{X}=\left.\left(\left.u\right|_{*}\right)^{*} u\right|_{X}$, where $u: I d \rightarrow * *$ is the Drinfeld isomorphism attached to $\beta$ (i.e. $\left.u\right|_{X}=$ $\left(\operatorname{ev}_{X} \beta_{X X^{*}} \otimes 1_{X^{* *}}\right)\left(1_{X} \otimes \operatorname{coev}_{X^{*}}\right)$, where ev, coev denote the evaluation and coevaluation morphisms).

Theorem 4.1. Let $\mathcal{C}$ be a finite rigid tensor category. If $\beta$ is a braiding on $\mathcal{C}$ then for any $X, Y \in \mathcal{C}$ the automorphism $\beta_{X Y}^{2}$ is quasiunipotent, i.e. $\left(\beta_{X Y}^{2}\right)^{N}$ is unipotent for some positive integer $N$. Similarly, the Casimir automorphism $z_{X}$ is quasiunipotent.

Proof. Let us compute determinants of automorphisms in $\mathcal{C}$ with respect to the dimension function $d_{+}$. From hexagon relation it follows that $\operatorname{det}\left(\beta_{X \otimes Y, Z}^{2}\right)=$ $\operatorname{det}\left(\beta_{X Z}^{2}\right)^{d_{+}(Y)} \operatorname{det}\left(\beta_{Y Z}^{2}\right)^{d_{+}(X)}$. Taking $Y=R$, and using that $X R=d_{+}(X) R$, we derive from this that $\operatorname{det}\left(\beta_{X Z}^{2}\right)^{d_{+}(R)}=1$. (Note that $\operatorname{det}\left(\beta_{Y Z}^{2}\right)$ makes sense for any element $Y$ of $\left.\mathbb{A} \otimes_{\mathbb{Z}} \mathcal{G}(\mathcal{C})\right)$.

Let us evaluate the determinant of identity (1) in $X \otimes R$, where $X$ is simple. We find, after cancelations:

$$
\operatorname{det}\left(\left.z\right|_{X}\right)^{d_{+}(R)}=\operatorname{det}\left(\beta_{X R}^{2}\right)^{-2}
$$

Raising this to power $d_{+}(R)$, we get $\operatorname{det}\left(\left.z\right|_{X}\right)^{d_{+}(R)^{2}}=1$. But $z$ is a scalar, so $\left(\left.z\right|_{X}\right)^{d_{+}(X) d_{+}(R)^{2}}=1$. Hence, $\left.z\right|_{X}$ is a root of unity, and thus $z^{L}$ is unipotent on all objects for some $L$. Taking $N=2 L$, we find that $\left(\beta_{X Y}^{2}\right)^{N}$ is unipotent for any $X, Y$.

\section{Refinement of Vafa's theorem for MTC}

Let now $\mathcal{C}$ be a modular tensor category (i.e, it is a semisimple ribbon category with a non-degenerate s-matrix). In this case, one has the automorphism of the identity functor $\theta$ called the twist, such that $\theta_{X \otimes Y}=\left(\theta_{X} \otimes \theta_{Y}\right) \beta_{X Y}^{-2}$ (it satisfies $\theta^{2}=z$ ). Vafa's theorem (see $[\mathrm{V}, \mathrm{AM}, \mathrm{BaKi}]$ ) claims that $\theta$ has finite order. Here we prove a refinement of this result, making an estimate on this order.

Let $D$ be the global dimension of $\mathcal{C}$, i.e. the sum of squares of categorical dimensions of its simple objects. It is a positive algebraic integer (see [BaKi]), which is always positive (see [ENO]).

Theorem 5.1. The order of $\theta$ is finite and divides $D^{5 / 2}$. 
Proof. Let $d(X), X \in \mathcal{C}$, be the categorical dimension of $X$. Then $d$ is a dimension function on $\mathcal{G}(\mathcal{C})$. Define the corresponding regular object $R$ by $R=\sum_{X \in S} d(X) X$. It is clear that $d(R)=D$. Let us argue as in the proof of Theorem 4.1. We find that $\operatorname{det}\left(\beta_{X Y}^{2}\right)^{D}=1$. Now, taking the determinant of the equation $\theta_{X \otimes Y}=\left(\theta_{X} \otimes \theta_{Y}\right) \beta_{X Y}^{-2}$ for $Y=R$, and raising it to power $D$, we get $\theta_{X}^{d(X) D^{2}}=1$. But by Lemma 1.2 in [EG2] (see also [BaKi]), $d(X)$ divides $D^{1 / 2}$. Thus, $\theta_{X}^{D^{5 / 2}}=1$ for any $X$, as desired.

Remark. The power $5 / 2$ cannot be replaced with anything less than 2 : for the category of representations of $\widehat{s l}_{2}$ at level 1 , one has $D=2$, while the twist of the nontrivial object is $\pm i$.

Recall now that any modular tensor category has a central charge, a number $c$ defined modulo 8 .

Corollary 5.2. Let $N$ be the number of simple objects of a modular tensor category $\mathcal{C}$, and $D$ its global dimension. Then $c N D^{5 / 2} / 2$ is an algebraic integer.

This refines Vafa's result that $c$ is rational.

Proof. Associated to $\mathcal{C}$ are the matrices $S, T$ satisfying the equations $S^{2}=C$ (the charge conjugation matrix), and $(S T)^{3}=C e^{\pi i c / 4}$. Since $\operatorname{det}(C)= \pm 1$, taking the determinants of these relations, we get $e^{\pi i c N}=\operatorname{det}(T)^{12}$. But the order of $\operatorname{det}(T)$ divides $D^{5 / 2}$, since its eigenvalues are the twists. This implies the result.

\section{Quasi-exponent of a tensor category}

The above results and the work [EG3] motivate the following definition: the quasiexponent $q \exp (\mathcal{C})$ of a finite rigid tensor category $\mathcal{C}$ is the smallest power $N$ such that $\left(\beta^{2}\right)^{N}$ is unipotent in the Drinfeld's center $Z(\mathcal{C})$.

Proposition 6.1. If $\mathcal{C}=\operatorname{Rep}(\mathrm{H})$, where $H$ is a finite dimensional Hopf algebra, then $\operatorname{qexp}(\mathcal{C})$ coincides with the quasiexponent of $H$ defined in [EG3].

Proof. By definition, the quasi-exponent of $H$ is the smallest $n$ such that $u^{n}$ is unipotent, where $u$ is the Drinfeld element of the double $D(H)=H \otimes H^{* c o p}$. On the other hand, the quasi-exponent of $\operatorname{Rep}(\mathrm{H})$ is the smallest $m$ such that $\left(R^{21} R\right)^{m}$ is unipotent, where $R$ is the universal R-matrix of $D(H)$.

Recall that $\Delta(u)=(u \otimes u)\left(R^{21} R\right)^{-1}$. Thus $m$ is a divisor of $n$, and it remains to show that if $\left(R^{21} R\right)^{m}$ is unipotent then so is $u^{m}$. This is proved similarly to [EG1], where this is done in the semisimple case.

Recall that we can write $u$ canonically as a product $u=u_{s s} u_{u n}$ of commuting semisimple and unipotent elements. Then we have

$$
\Delta\left(u_{s s}^{m}\right) \Delta\left(u_{u n}^{m}\right)=\left(u_{s s}^{m} \otimes u_{s s}^{m}\right) \cdot\left(\left(u_{u n}^{m} \otimes u_{u n}^{m}\right)\left(R^{21} R\right)^{-m}\right)
$$

Note that $u_{s s} \otimes u_{s s}$ and $u_{u n} \otimes u_{u n}$ commute with $R^{21} R$, since so does $u \otimes u$. Hence the two sides of equation (2) are two decompositions of the same element into a 
product of commuting semisimple and unipotent parts. So these decompositions must coincide, which means that $u_{s s}^{m}$ is a grouplike element.

By Radford's theorem [R], $u_{s s}^{m}=a b$, where $a, b$ are commuting in $D(H)$ grouplike elements of $H$ and $H^{*}$, respectively. Thus, we find that $a^{-1} b^{-1} u^{m}$ is unipotent, and all factors in this product commute.

Now the proof is finished by the following lemma, which is a straightforward generalization of Proposition 3.2 in [EG3]:

Lemma 6.2. Let $g \in H, \alpha \in H^{*}$ be grouplike elements such that $\alpha u^{m} g$ is unipotent for some $m$. Then $g=\alpha=1$.

Proof. The proof is almost identical to the proof of Proposition 3.2 of [EG3] (which treats the case $\alpha=1$ ). We have $\left(1-\alpha u^{m} g\right)^{N}=0$ for some integer $N>0$. This is equivalent to

$$
\sum_{k=0}^{N}(-1)^{k}\left(\begin{array}{c}
N \\
k
\end{array}\right) \alpha^{k} u^{m k} g^{k}=0 .
$$

This, in turn, is equivalent to

$$
\sum_{k=0}^{N}(-1)^{k}\left(\begin{array}{c}
N \\
k
\end{array}\right) R_{m k}\left(g^{k} \otimes \alpha^{-k}\right)=0,
$$

where $R_{l}:=R\left(1 \otimes S^{2}\right)(R) \ldots\left(1 \otimes S^{2 l-2}\right)(R)$. Indeed, equation (3) is obtained from equation (4) by applying the antipode in the second component, and then multiplying the second component by the first component. So the equivalence of the two equations follows from the fact that the multiplication map $H^{*} \otimes H \rightarrow$ $D(H)$ is a linear isomorphism.

Now, apply $1 \otimes \varepsilon$ to equation (4). We get $\sum_{k=0}^{N}(-1)^{k}\left(\begin{array}{c}N \\ k\end{array}\right) g^{k}=0$, i.e., $(1-g)^{N}=0$. However, $1-g$ is semisimple as $g$ has finite order, hence $g=1$. Similarly, applying $\varepsilon \otimes 1$ to (4), we get $\alpha=1$.

Proposition 6.1 is proved.

\section{Proposition 6.3.}

1) $q \exp (\mathcal{C})=q \exp (Z(\mathcal{C}))$

2) If $\mathcal{C}$ is semisimple, $q \exp (\mathcal{C})=\operatorname{qexp}\left(\mathcal{C}^{*}\right)$, where $\mathcal{C}^{*}$ is the dual category ${ }^{1}$ of $\mathcal{C}$ with respect to an indecomposable module category. ${ }^{2}$

3) If $\mathcal{C}$ is semisimple and $\operatorname{qexp}(\mathcal{C})=n$ the $\left(\beta^{2}\right)^{n}=1$.

Proof.

- 1) follows from the fact that $Z(Z(\mathcal{C}))=Z(\mathcal{C}) \otimes Z(\mathcal{C})$ as braided categories.

\footnotetext{
${ }^{1}$ About dual categories, see e.g. [ENO].

${ }^{2}$ Thus, quasi-exponent is a Morita invariant of tensor categories in the sense of Müger.
} 
- 2) We claim that $Z\left(\mathcal{C}^{*}\right)$ is equivalent to $Z(\mathcal{C})$ with opposite braiding, as braided categories. Indeed, one may assume that $\mathcal{C}=\operatorname{Rep}(H)$, where $H$ is a weak Hopf algebra, and $\mathcal{C}^{*}=\operatorname{Rep} H^{* c o p}$ (see [ENO]). Then $Z(\mathcal{C})=$ $\operatorname{Rep}(D(H)), Z\left(\mathcal{C}^{*}\right)=\operatorname{Rep} D\left(H^{* c o p}\right)$. But $D(H)$ is isomorphic to $D\left(H^{* c o p}\right)$, in such a way that the $R$-matrix of $D(H)$ maps to the inverse of opposite $R$-matrix of $D\left(H^{* c o p}\right)$. Thus, 1) implies 2$)$.

- To prove 3 ), it suffices to observe that identity (1) implies that $\beta^{2}$ is a semisimple operator on $\operatorname{Hom}(U, X \otimes Y)$ for any simple objects $X, Y, U$ of $Z(\mathcal{C})$ (its square is a scalar).

Remark. In view of Proposition 6.3(3), in the semisimple case the quasiexponent of $\mathcal{C}$ should be called the exponent of $\mathcal{C}$ (by analogy with [EG1]).

\section{Acknowledgements}

I thank A. Davydov for very useful discussions which inspired this note, and S. Gelaki for collaboration in [EG1], where the methods used here were introduced. I am grateful to MPIM (Bonn) for hospitality. This research was partially supported by the NSF grant DMS-9988796, and was done in part for the Clay Mathematics Institute.

\section{References}

[AB] Arad, Z., Blau, H., On table algebras and applications to finite group theory, J. Algebra 138 (1991), no. 1, 137-185.

[AM] G. Anderson, G. Moore, Rationality in conformal field theory, Comm. Math. Phys. 117 (1988), 441-450.

[BaKi] B. Bakalov, A. Kirillov Jr., Lectures on Tensor categories and modular functors, University Lecture Series, 21. American Mathematical Society, Providence, RI, 2001.

[Da1] A. Davydov, K-theory of braided monoidal categories, MPI preprint, 2001; http://www.mpim-bonn.mpg.de/cgi-bin/preprint/

[Da2] - Finite groups with the same character tables, Drinfeld algebras and Galois algebras, Algebra (Moscow, 1998), 99-111, de Gruyter, Berlin, 2000.

[EG1] P. Etingof, S. Gelaki, On the exponent of finite-dimensional Hopf algebras, Math. Res. Lett. 6 (1999), 131-140.

[EG2] S Some properties of finite-dimensional semisimple Hopf algebras, Math. Res. Lett. 5 (1998), 191-197.

[EG3] - On the quasi-exponent of finite-dimensional Hopf algebras, Math. Res. Lett. 9 (2002), 277-287.

[ENO] P. Etingof, D. Nikshych, V. Ostrik, On fusion categories, preprint; math.QA/0203060

[FK] J. Fröhlich, T. Kerler, Quantum groups, quantum categories and quantum field theory, Lecture Notes in Mathematics, 1542. Springer-Verlag, Berlin, 1993.

[R] D. Radford, Minimal quasitriangular Hopf algebras, J. Algebra 157 (1993), 285-315.

[V] C. Vafa, Toward classification of conformal theories, Phys. Lett. B 206 (1988), 421426.

Department of Mathematics, Massachusetts Institute of Technology, Cambridge, MA 02139, U.S.A.

E-mail address: etingof@math.mit.edu 